\title{
Temperature Dependence Analysis of Mode Dispersion in Step-Index Polymer Optical Fibers
}

\author{
M. KovACEVIC ${ }^{a, *}$ AND A. DJORDJEVICH ${ }^{b}$ \\ ${ }^{a}$ Faculty of Science, Department of Physics, University of Kragujevac, Serbia \\ ${ }^{b}$ City University of Hong Kong, Tat Chee Avenue, Kowloon, Hong Kong
}

Temperature dependence of the mode dispersion is investigated for commercially available polymethyl methacrylate (PMMA) based step-index polymer optical fibers. An analytical expression is proposed describing the thermal variation of the fiber refractive index. This index decreases with increasing temperature as density of the polymer material drops. The study covered the temperature range from $-60{ }^{\circ} \mathrm{C}$ to $100{ }^{\circ} \mathrm{C}$. Results show that the modal dispersion decreases and the bandwidth increases with rising temperature.

PACS numbers: 42.81.-i, 42.81.Ht, 42.81.Dp

\section{Introduction}

Polymer optical fiber (POF) has attracted considerable attention particularly for sensing and short-distance communication in local area networks. Compared to conventional glass optical fibers, POF provides the advantages of large core diameters, ductility, lightweight, and ease of connection. The main types of POFs, their manufacture, and possible present and expected future applications have been reported [1-3].

While much research has focused on the computational simulation of POF performance [4-7], few publications report on the influence of temperature on optical characteristics of large-core step-index POF [8-10]. Chen et al. [9]. investigated temperature effects on power attenuation in deformed POFs. Measurement of thermal variations of refractive index of polymethyl methacrylate (PMMA), polystyrene, and poly (4-methyl-pentene) have also been reported [10]. Little has been published on mode dispersion and bandwidth in step-index POFs under extreme temperature conditions.

This paper extends the work presented in [10] to obtain an analytical function that describes the refractive index variation with temperature for large-core PMMA fibers. The modal dispersion and bandwidth are calculated within a wide range of temperatures, varying from -60 to $+100^{\circ} \mathrm{C}$.

\section{Theoretical approach}

Transmission of information along optical fibers is normally achieved by sending out a sequence of light pulses. However, as an individual pulse propagates, it spreads

\footnotetext{
* corresponding author; e-mail: kovac@kg.ac.rs
}

out due to dispersive fiber properties, which causes the pulse to broaden or spread along the temporal axis. Three dispersion types have been identified: modal dispersion (sometimes referred to as ray dispersion), material dispersion, and waveguide dispersion. Fundamentals about this topic, presented simply and illustrated by numerous examples, can be found in paper by Snyder and Love [11]. Because commercial POFs are step-index fibers at present, modal dispersion dominates dispersion in high bit-rate POF networks. The influence of the other two dispersion types is negligible in comparison. In regard to the polymer fibers dealt with in this paper only mode dispersion plays a role so that we deal solely with this effect.

Since the light paths within the fiber can have different lengths, modes that have started simultaneously (as part of one pulse) arrive at different times at the fiber's output, contributing thus to pulse broadening. Simultaneously launched modes will therefore reach the output end over the time interval $\Delta \tau$ :

$$
\Delta \tau=\frac{n_{1} L}{c}\left(\frac{n_{1}}{n_{2}}-1\right)=\frac{L}{2 c n_{2}} A_{\mathrm{N}}^{2},
$$

where $n_{1}$ and $n_{2}\left(<n_{1}\right)$ represent respectively the refractive indices of core and cladding; $c$ represents the velocity of light in the free space and $A_{N}=\sqrt{n_{1}^{2}-n_{2}^{2}}$ is numerical aperture; $L$ is the fiber's length. It is evident from (1) that the greater the fiber length, the greater the pulse spreading results. For a weakly guiding fiber when $n_{1} \cong n_{2}$, using $\Delta=\left(n_{1}-n_{2}\right) / n_{2}$, it is obtained

$$
\Delta \tau \cong \frac{L n_{1}}{c} \Delta \cong \frac{L n_{1} \theta_{z(\max )}}{2 c},
$$

where $\theta_{z(\max )}$ represents the complement of the critical angle, defined by $\theta_{z(\max )}=\cos ^{-1}\left(n_{2} / n_{1}\right)$. Knowledge of the mode dispersion alone is not sufficient for estimating the actual information carrying capacity of the 
optical fibers. Bandwidth plays a part. The maximum unrepeatred link length is imposed by fiber attenuation and dispersion. A commonly used criterion for maximum allowed pulse dispersion can be found in [12] in the following form:

$$
\Delta \tau \leq T_{\mathrm{B}} / 4,
$$

where $T_{\mathrm{B}}$ is the bit duration. In terms of the bit rate $B$ $\left(=1 / T_{\mathrm{B}}\right)$, Eq. (3) can be rewritten as

$$
4 \Delta \tau B \leq 1 \text {. }
$$

The consequence of this broadening is that the time gap between the bits becomes smaller. Excessive spreading causes the communication bits to "overflow" their intended time slots and overlap with adjacent ones. The receiver may then have difficulty discerning and interpreting adjacent bits, which increases the bit error rate.

The product of bandwidth and length characterizes the transmission capacity of a fiber. For the Gaussian-shaped pulses [2]:

$$
B \cdot L \approx \frac{0.44}{\Delta \tau} L
$$

where $L$ represents the fiber's length. In POF systems, the limiting factor is usually the bandwidth of the fiber itself, which is created by modal dispersion. The following general relationship can be used as a guide for digital systems [2]:

maximum bit rate $[\mathrm{Mbit} / \mathrm{s}]=2 \times$ bandwidth $[\mathrm{MHz}]$.

For a typical step-index POF, $n_{1}=1.492$ and $\Delta=$ $\left(n_{1}-n_{2}\right) / n_{2} \approx 0.06$. Using Eq. (6), for $L=100 \mathrm{~m}$, a delay of $\Delta \tau \simeq 30 \mathrm{~ns}$ is obtained. Thus, in accordance with criteria (4), two pulses separated by $100 \mathrm{~ns}$ at the input end may be resolvable at the end of a $100 \mathrm{~m}$ long fiber but not at the end of twice as long fiber as the delay would then be 60 ns.

\section{Simulation results and discussion}

In general, polymers are extremely sensitive to environmental conditions such as temperature. Based on the previously reported experimental results [10], we have analyzed the refractive index variation with temperature for large-core PMMA POF. By a nonlinear least square fit, the following function is proposed for the variation of the refractive index: $n(T)=n_{0}+a T+b T^{2}$ where $n_{0}=1.49538$. The coefficients $a$ and $b$ attest to the near-linear nature of this dependence with temperature. Their values of $a=-0.000115\left({ }^{\circ} \mathrm{C}\right)^{-1}$ and $b=$ $-5 \times 10^{-7}\left({ }^{\circ} \mathrm{C}\right)^{-2}$ have been obtained. Using this function, the refractive index calculated at $20^{\circ} \mathrm{C}$ is 1.49287 , which is consistent with the usual values for the material in question. Temperature dependence of refractive index is shown in Fig. 1.

Figure 2 shows the modal dispersion of the PMMA step-index POF as a function of both, fiber's length and temperature. The assumption was that the core refractive index variation with temperature mostly contributes to the temperature dependence of mode dispersion. The modal dispersion of PMMA increases linearly

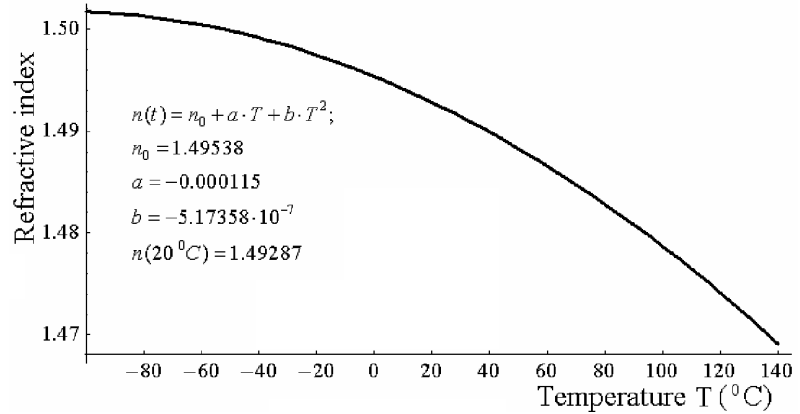

Fig. 1. Thermal variation of refractive index of PMMA based step-index POF.

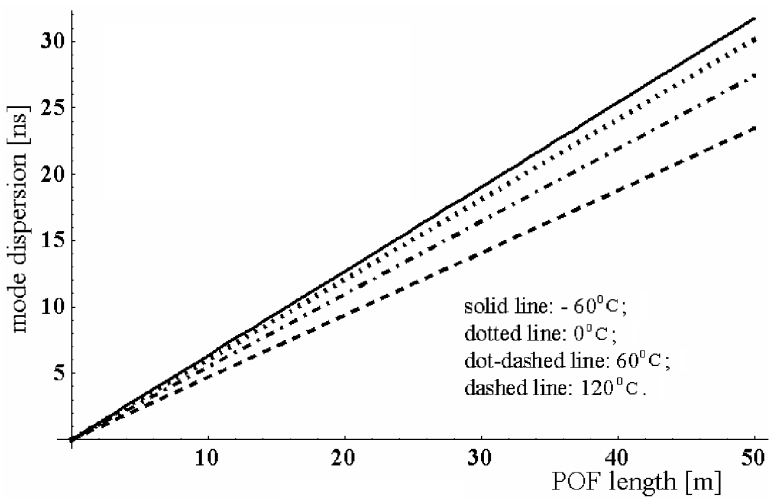

Fig. 2. Mode dispersion versus length of fiber for four different temperatures.

with length. The modal dispersion decreases as temperature increases. It is obvious that the slope of the linear functions, $\tau=\tau(L)$ decreases as temperature increases. Hence from (2), one can obtain the variation of pulse delay with temperature

$$
\frac{\mathrm{d} \tau}{\mathrm{d} T}=\frac{L \Delta}{c} \frac{\mathrm{d} n_{1}}{\mathrm{~d} T} \text {. }
$$

We also calculated temperature dependence of band-

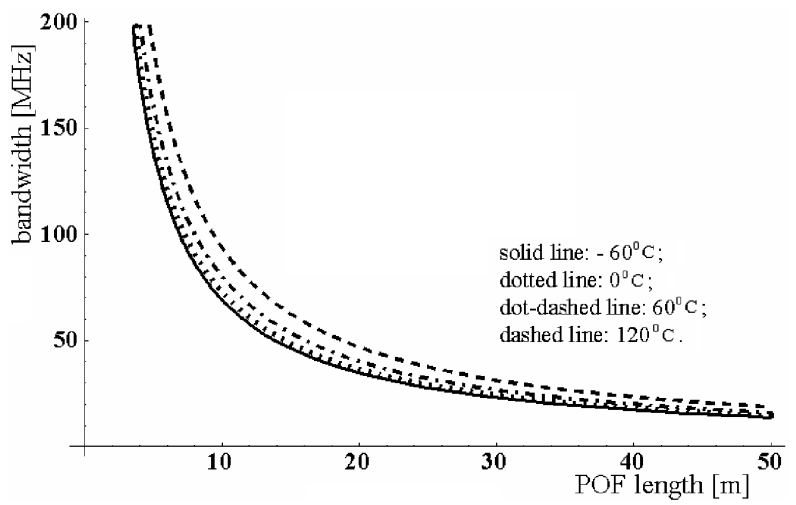

Fig. 3. Bandwidth of PMMA step-index POF versus fiber length for four different temperatures. 
width for PMMA step-index POF. Figure 3 shows the bandwidth for PMMA based POF as function of fiber length and temperature, respectively. The calculated bandwidth is strongly dependent on fiber length. Although the bandwidth difference with temperature decreases with the increasing fiber length, it remains clearly evident even after $50 \mathrm{~m}$.

\section{Conclusion}

The influence of temperature on the refractive index was studied for commercially available large-core PMMA POFs. An analytical function of thermal variation of refractive index with temperature has been proposed. Refractive index decreases with increasing temperature because of the drop in the density of the polymer material. The modal dispersion related to the refractive index of the fiber core was also calculated and presented. As a summary of the results obtained, we found that the modal dispersion decreases with temperature at constant $\Delta$, and that the bandwidth increases slightly with the increase of temperature.

\section{Acknowledgments}

The work described in this paper was supported by a grant from City University of Hong Kong (SRG Project No. 7002313) and in part by Serbian Ministry of Science, through Project No. 141023.

\section{References}

[1] J. Zubia, J. Arrue, Opt. Fiber Technol. 7, 101 (2001).

[2] W. Daum, J. Krauser, E.P. Zamzow, O. Zeimann, POF-Polymer Optical Fibers for Data Communication, Springer-Verlag, Berlin 2002.

[3] POF Modelling Theory, Measurement and Application, Eds. C.A. Bunge, H. Poisel, Books on Demand, Norderstedt 2007.

[4] M.S. Kovacevic, D. Nikezic, A. Djordjevich, Appl. Opt. 44, 3898 (2005).

[5] A.F. Garito, J. Wang, R. Gao, Science 281, 962 (1998).

[6] G. Aldabaldetreku, G. Durana, J. Zubia, J. Arrue, J. Light. Technol. 24, 3770 (2006).

[7] G. Durana, G. Aldabaldetreku, J. Zubia, J. Arrue, C. Tanaka, Opt. Express 16, 7929 (2008).

[8] A. Priyadarashi, L. Shimin, E.H. Wong, R. Rajoo, S.G. Mihaisalkar, V. Kirpesh, J. Electron. Mater. 34, 1378 (2005).

[9] L.W. Chen, W.H.Y.C. Lu, Chen, Opt. Commun. 2009 in press.

[10] P. Michel, J. Dugas, J.M. Cariou, L. Martin, J. Macromol. Sci. Phys. B 25, 379 (1986).

[11] A. Snyder, J.D. Love, Optical Waveguide Theory, Champan and Hall, London 1983.

[12] A. Ghatak, K. Thyagarajan, Introduction to Fiber Optics, Cambridge University Press, Cambridge 1998. 